\title{
Chemoenzymatic method to enantiopure sulphur heterocyclic $\beta$-hydroxy nitriles
}

\author{
Mihaela C. Turcu, Päivi Perkiö, and Liisa T. Kanerva* \\ Department of Pharmacology, Drug Development and Therapeutics/Laboratory of Synthetic \\ Drug Chemistry and Department of Chemistry, University of Turku, Lemminkäisenkatu 5 C, \\ FIN-20520, Turku, Finland \\ E-mail: lkanerva@utu.fi
}

\section{Dedicated to Professor Harri Lönnberg on the occasion of his $60^{\text {th }}$ birthday}

\begin{abstract}
Sulphur heterocyclic $\beta$-hydroxy nitriles are prepared from the suitable aldehyde and (trimethylsilyl)acetonitrile in the presence of lithium acetate at good yields. Burkholderia cepacia lipase allowed the formation of the both enantiomers of $\beta$-hydroxy nitriles in highly enantiopure forms (ee 99\%) through acylation of a racemic mixture and alcoholysis of the acylated enantiomer in tert-butyl methyl ether.
\end{abstract}

Keywords: Nitriles, $(R)$ - and $(S)$ - $\beta$-hydroxy nitriles, lipase catalysis, kinetic resolution

\section{Introduction}

The enantiomers of $\beta$-hydroxy nitriles possess high potential as intermediates in organic synthesis and medicinal chemistry. This is due to the easy transformation of the cyano group into other functionalities, leading to the formation of $\beta$-hydroxy acids, their derivatives and $\gamma$-amino alcohols. As a result, synthetic methods to access both racemic and enantiopure $\beta$-hydroxy nitriles are continuously improved.

Anti-depressants with a 3-amino-1-arylpropan-1-ol structure are widely used for the treatment of psychiatric and metabolic disorders. Chiral $\beta$-hydroxy nitriles are convenient intermediates for their synthesis. When a $\beta$-hydroxy nitrile motif is attached to a heteroaromatic scaffold a broad range of potential biologically active compounds can be prepared. An illustrative example is $(S)$-duloxetine, ${ }^{1}$ a $(S)$-3-amino-1-(2-thienyl)propan-1-ol-based serotoninnorepinephrine reuptake inhibitor for treating diabetic neuropathy, depression and anxiety. ${ }^{2}$ In this context the need for reliable methods to access the enantiomers of new sulphur containing $\beta$ hydroxy nitriles is understandable. Herein we describe the chemoenzymatic syntheses of the both 
enantiomers of new sulphur heterocycles 1(b-f) (Scheme 1). Compound 1a in enantiopure forms is known and serves as a reference compound for our work. ${ }^{1} \mathbf{1 c}$ is used as a model substrate for optimization. Lipase-catalyzed asymmetric acylation is the enzymatic step used for enantioseparation while lipase-catalyzed alcoholysis is used to transform the acylated enantiomers $(R)$-2(a-e) back into the corresponding $\beta$-hydroxy nitrile enantiomers under mild conditions. The study is continuation to the previous works in the group with sulphur heterocyclic aldehydes as starting materials where dynamic kinetic resolution (DKR) methods by lipases have been developed in the formation of $\alpha$-hydroxy nitriles (cyanohydrins). ${ }^{3 a, b}$

Early biocatalytic attempts to access enantiopure $\beta$-hydroxy nitriles have been based on the baker's yeast reduction of the corresponding $\beta$-keto nitriles. ${ }^{4}$ The first lipase-catalyzed access consisted of the hydrolysis of the esters of $\beta$-hydroxy nitriles. ${ }^{5 a, b}$ Kamal's group has used lipasecatalyzed transesterification for the kinetic resolution of 3-hydroxy-3-phenylpropanenitriles ${ }^{6 a, b}$ and 4-aryloxy-3-hydroxybutanenitriles. ${ }^{7}$ Bäckvall's group combined lipase catalysis with $\mathrm{Ru}-$ complex catalysis and developed DKR to prepare enantiopure $\beta$-hydroxy nitriles. ${ }^{8 \mathrm{a}, \mathrm{b}}$ Previously, also racemic $\beta$-hydroxy nitriles have been enantioselectively hydrolyzed into the corresponding enantiopure acids and amides with satisfactory enantioselectivity using microbial whole cells containing nitrile hydratase/amidase activity. ${ }^{9}$

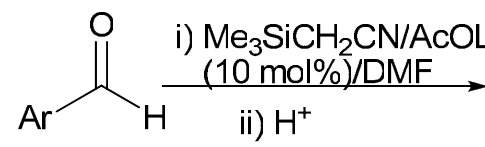

ii) $\mathrm{H}^{+}$

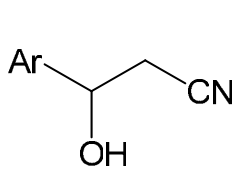

rac-1(a-f)

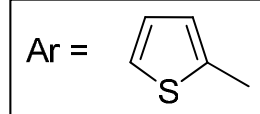

rac-1a<smiles>Cc1cc2ccccc2s1</smiles>

rac-1d

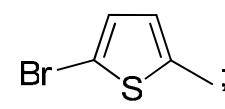

rac-1b

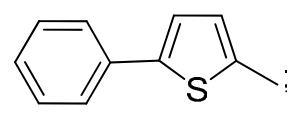

rac-1c<smiles>Cc1csc2ccccc12</smiles>
rac-1e

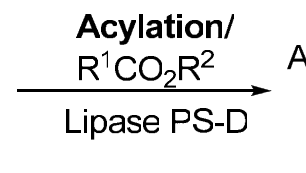<smiles>[R]O[C@@H]([IH])CCl</smiles>

$(R)-2(a-f)$

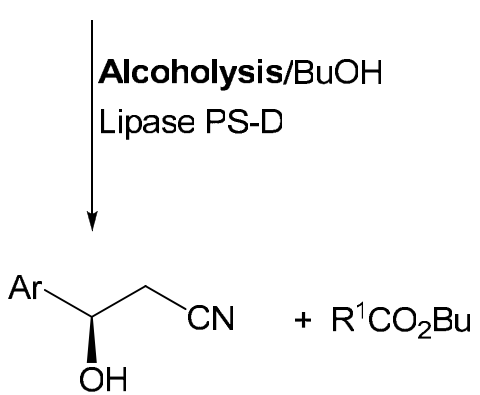

$(R)-1(\mathbf{a}-\mathbf{f})$

Scheme 1

\section{Results and Discussion}

\section{Chemical synthesis of racemates}

Basically, two main strategies are available for the preparation of $\beta$-hydroxy nitriles: one exploits the ring opening of epoxides with cyanides from various sources as nucleophiles and the other 
the addition of deprotonated nitriles to carbonyl compounds, most often to aldehydes. ${ }^{10,11,12} \mathrm{We}$ chose the aldehyde route for the preparation of $\beta$-hydroxy nitriles $r a c-1(a-f)$ because various sulphur-containing heterocyclic aldehydes are readily commercially available. Nowadays, alkali acetonitriles as cyanide sources in condensation with aldehydes have been more and more often replaced by (trimethylsilyl)acetonitrile $\left(\mathrm{TMSCH}_{2} \mathrm{CN}\right)$ in order to get satisfactory yields of $\beta$ hydroxy nitriles. ${ }^{10,13}$ Accordingly, we followed the previous procedure ${ }^{13}$ and prepared rac-1(a-f) through the cyanomethylation of aldehydes with $\mathrm{TMSCH}_{2} \mathrm{CN}$ catalyzed by lithium acetate as a Lewis base. The isolated yields of the products were usually higher than $80 \%$ as described in the Experimental Section. As exceptions, rac-1d was obtained at $65 \%$ and $r a c-1$ only at $41 \%$ chemical yields.

Acetate $(r a c-2(\mathbf{a}-\mathbf{d}))$ and butanoate ( $r a c-2 e)$ esters were prepared from the corresponding anhydrides and rac-1(a-e) by standard procedure.

Table 1. Lipase screening $\left(50 \mathrm{mg} \mathrm{mL}^{-1}\right)$ for the acylation of $\mathrm{rac}-\mathbf{1 c}(0.1 \mathrm{M})$ with vinyl acetate $(0.2 \mathrm{M})$ in TBME

\begin{tabular}{clccccc}
\hline Entry & \multicolumn{1}{c}{ Enzyme } & $\begin{array}{c}\text { Time } \\
(\mathrm{h})\end{array}$ & $\begin{array}{c}\text { Conversion } \\
(\%)\end{array}$ & $\begin{array}{c}\mathrm{ee}^{(R)-2 \mathrm{c}} \\
(\%)\end{array}$ & $\begin{array}{c}\mathrm{ee}^{(S)-1 \mathrm{c}} \\
(\%)\end{array}$ & $E$ \\
\hline 1 & Lipase PS-D & 0.5 & 50 & 99 & 98 & $» 200$ \\
2 & Lipase PS-C II & 0.5 & 46 & 96 & 82 & 113 \\
3 & Lipase AK-C & 0.5 & 48 & 99 & 94 & $» 200$ \\
4 & CAL-A & 3 & 60 & 4 & 7 & 1 \\
5 & CAL-B & 50 & 48 & 94 & 90 & 108 \\
6 & Lipozyme RM IM & 72 & 2 & 99 & 2 & $» 200$ \\
7 & Lipozyme TL IM & 24 & 43 & 96 & 72 & 117 \\
\hline
\end{tabular}

\section{Enzymatic acylation}

Lipase-catalyzed kinetic resolution was optimized using rac-1c as a model compound. Commercially available lipases from Pseudomonas cepacia (lipase PS-D and lipase PS-C II), Pseudomonas fluorescens (lipase AK-C), Rhizomucur miehei (Lipozyme RM IM) and Thermomyces lanuginosus (Lipozyme TL IM) as well as from Candida antarctica lipases A (CAL-A) and B (CAL-B) were first screened for transesterification of rac-1c in tert-butylmethyl ether (TBME) using vinyl acetate as an irreversible acyl donor. As shown in Table 1, enantioselectivity as measured using enantiomeric ratio $E$ is excellent ( $E \gg 200$; exact $E$ values are not given due to extensive effects caused by even minor experimental inaccuracies when $E$ is high) in the cases of lipase PS-D, AK-C and Lipozyme RM IM (entries 1, 3 and 6). Taking also conversion and time into account it is clear that lipase PS-D is the most effective of the studied catalysts. Accordingly, the acylation tends to stop at $50 \%$ conversion when the reactive $R$ enantiomer of the initially racemic mixture has reacted (entry 1). The Kamal group also found lipase PS-D the most appropriate for the kinetic resolution of $\beta$-hydroxy nitriles including the highly enantioselective $R$-acylation of $r a c-1$ a in diisopropyl ether (DIPE). ${ }^{1,6 a, b, 7,10}$ Lipase PS-D 
was a natural choice for further studies, and it was expected to show $R$-enantioselectivity in accordance with the known $R$-selectivity for the acylation of $\mathrm{rac}-\mathbf{1 a}$.

Next rac-1c was subjected to acylation with vinyl acetate varying the lipase PS-D content from 50 through 25 and 10 to $5 \mathrm{mg} \mathrm{mL}^{-1}$ in TBME. The enzyme content had not an effect on enantioselectivity ( $E \gg 200$ was achieved) whereas conversions reached after 15 minutes dropped from 50 through 42 and 27 to $14 \%$ with the given decreasing enzyme contents. In the presence of $25 \mathrm{mg} \mathrm{mL}^{-1}$ of lipase PS-D with $42 \%$ conversion after 15 minutes TBME was shown to be the best solvent as the reaction in DIPE (conversion 37\%) and toluene (conversion 36\%) took place slower. Moreover, minor solubility problems of rac-1c were linked to DIPE and toluene.

When an acyl donor $\mathrm{R}^{1} \mathrm{CO}_{2} \mathrm{R}^{2}$ reacts with a racemic alcohol like rac-1c in the formation of an ester like $(R)$-2c (Scheme 1$) \mathrm{R}^{2} \mathrm{OH}$ is liberated. For a successful kinetic resolution, it is important that the acyl transfer is irreversible, i.e., that the enzymatic reaction of $\mathrm{R}^{2} \mathrm{OH}$ with $(R)$ 2c is prevented. Finally, the most common irreversible acyl donors were screened for the acylation of rac-1c in TBME using $25 \mathrm{mg} \mathrm{mL}^{-1}$ of lipase PS-D (Table 2). It is worth to remember that 2,2,2-trifluoroethyl butanoate is not necessarily irreversible as an acyl donor although it is generally expected to be as the nucleophilic character of the liberated 2,2,2trifluoroethanol is low and it is not reactive when added as such to the resolution mixture. Thus, interesterification between an amino ester and 2,2,2-trifluoroethyl esters was previously shown to be highly effective in the presence of lipase PS and CAL-B preparations. ${ }^{14 a, b}$ In the present work, the acylation of rac-1c proceeds in a highly enantioselective manner with the tested acyl donors while reactivity is excellent only in the case of vinyl esters (entries 1-4). Vinyl acetate as an acyl donor is somewhat more favourable over the butanoate (entry 3 compared to 4). Enhanced substrate concentration somewhat lowers reactivity (entry 1 compared to 2).

Table 2. Effect of acyl donors $(0.2 \mathrm{M})$ for the acylation of $\mathrm{rac}-1 \mathrm{c}(0.1 \mathrm{M})$ in the presence of lipase PS-D $\left(25 \mathrm{mg} \mathrm{mL}^{-1}\right)$ in TBME

\begin{tabular}{clccccc}
\hline Entry & \multicolumn{1}{c}{ Acyl donor } & $\begin{array}{c}\text { Time } \\
(\mathrm{h})\end{array}$ & $\begin{array}{c}\text { Conversion } \\
(\%)\end{array}$ & $\begin{array}{c}\mathrm{ee}^{(R)-2 \mathrm{c}} \\
(\%)\end{array}$ & $\begin{array}{c}\mathrm{ee}(S)-1 \mathrm{c} \\
(\%)\end{array}$ & $E$ \\
\hline 1 & $\mathrm{CH}_{3} \mathrm{CO}_{2} \mathrm{CH}=\mathrm{CH}_{2}{ }^{\mathrm{a}}$ & 0.25 & 37 & 99 & 58 & $» 200$ \\
2 & $\mathrm{CH}_{3} \mathrm{CO}_{2} \mathrm{CH}=\mathrm{CH}_{2}$ & 0.25 & 42 & 99 & 72 & $» 200$ \\
3 & $\mathrm{CH}_{3} \mathrm{CO}_{2} \mathrm{CH}=\mathrm{CH}_{2}$ & 0.5 & 50 & 99 & 94 & $» 200$ \\
4 & $\mathrm{PrCO}_{2} \mathrm{CH}=\mathrm{CH}_{2}$ & 0.5 & 42 & 99 & 71 & $» 200$ \\
5 & $\mathrm{PrCO}_{2} \mathrm{CH}_{2} \mathrm{CF}_{3}$ & 0.5 & 20 & 99 & 26 & $» 200$ \\
6 & $\mathrm{CH}_{3} \mathrm{CO}_{2} \mathrm{C}_{\left(\mathrm{CH}_{3}\right)=\mathrm{CH}_{2}}$ & 0.5 & 17 & 99 & 17 & $» 200$ \\
\hline
\end{tabular}

${ }^{\mathrm{a}} \operatorname{Rac}-1 \mathrm{c}(0.2 \mathrm{M})$ and vinyl acetate $(0.4 \mathrm{M})$

The set of $\beta$-hydroxy nitriles rac-1(a-f) was subjected to kinetic resolution with lipase PS-D in TBME (Table 3). Attention was paid to the amount of the enzyme needed to have a feasible process. Lipase PS-D possesses excellent enantioselectivity for all thiophen-based $\beta$-hydroxy 
nitriles rac-1(a-e) (entries 1-6) while enantioselectivity is only moderate in the case of thiazolbased rac-1f (Table 3, entry 7). As in the case of rac-1c (Table 2, entry 4) enzymatic reactivity for rac-1e with vinyl butanoate was low compared to the reaction with vinyl acetate as an acyl donor (data not shown). We achieved somewhat lower enantioselectivity for the lipase PS-Dcatalyzed acylation of rac-1a in TBME (Table 3, entry 1) compared to the previous acylation in DIPE (ee $>99 \%$ for both resolution products at $50 \%$ conversion). ${ }^{1}$

Table 3. Lipase PS-D catalyzed kinetic resolution of rac-1(a-f) $(0.1 \mathrm{M})$ with vinyl acetate $(0.2 \mathrm{M})$ in TBME

\begin{tabular}{llcccccc}
\hline Entry & Compound & $\begin{array}{c}\text { Amount of lipase } \\
\left(\mathrm{mg} \mathrm{mL}^{-1}\right)\end{array}$ & $\begin{array}{c}\text { Time } \\
(\mathrm{h})\end{array}$ & $\begin{array}{c}\text { Conversion } \\
(\%)\end{array}$ & $\begin{array}{c}\mathrm{ee}^{(R)-2(\mathrm{a}-\mathrm{f})} \\
(\%)\end{array}$ & $\begin{array}{c}\mathrm{ee}^{(S)-1(\mathrm{a}-\mathrm{f})} \\
(\%)\end{array}$ & $E$ \\
\hline 1 & $r a c-1 \mathbf{1 a}$ & 25 & 2 & 51 & 94 & 97 & 126 \\
2 & $r a c-1 b$ & 25 & 6 & 50 & 99 & 99 & $» 200$ \\
3 & $r a c-1 c$ & 10 & 1 & 48 & 99 & 91 & $» 200$ \\
4 & $r a c-1 c$ & 25 & 0.5 & 50 & 99 & 94 & $» 200$ \\
5 & $r a c-1 d$ & 25 & 3 & 50 & 99 & 99 & $» 200$ \\
6 & $r a c-1 e^{a}$ & 75 & 48 & 50 & 99 & 98 & $» 200$ \\
7 & $r a c-1 \mathbf{1 f}$ & 25 & 3 & 61 & 62 & 96 & 15 \\
\hline
\end{tabular}

${ }^{a}$ Vinyl butanoate $(0.2 \mathrm{M})$ was used as an acyl donor due to analytical problems in ee determination with the acetate of $\mathbf{1 e}$.

\section{Enzymatic alcoholysis}

It is natural that lipases catalyze the acylation of an alcohol with $\mathrm{R}^{1} \mathrm{CO}_{2} \mathrm{R}^{2}$ and the deacylation of the corresponding ester with an alcohol based on the same enantiopreference $(R$ in the present case as shown in Schemes 1 and 2). We investigated lipase PS-D-mediated alcoholysis primarily aiming to deacylate the $(R)$-esters 2 (a-e) obtained through the preparative scale asymmetric acylation under mild conditions as shown below. In addition, we wanted to know how enentioselective the alcoholysis is. Accordingly, each of rac-2(a-e) $(0.1 \mathrm{M})$ was subjected to alcoholysis with 1-butanol $(0.3 \mathrm{M})$ in TBME (Table 4). Alcohol content was kept as low as possible so that $(R)-\mathbf{1}(\mathbf{a}-\mathbf{e})$ was still completely formed because reactivity decreased with increasing alcohol concentrations. Alcohols are reported as competitive inhibitors to lipases. ${ }^{15 a, b}$ The usual enzyme content used was $50 \mathrm{mg} \mathrm{mL}^{-1}$ so that the alcoholysis smoothly proceeded to $50 \%$ conversion with excellent enantioselectivity (E»200). In the case of rac-2e very slow reactions were observed even at high enzyme contents and even at elevated temperature (entries $5-7)$. 


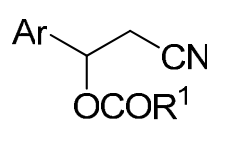

rac-2(a-e)

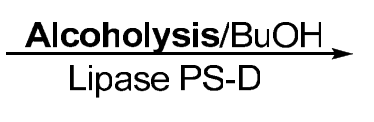

Lipase PS-D
$\widehat{\mathrm{OH}}_{\mathrm{CN}}^{\mathrm{Ar}}+\mathrm{NC}_{\mathrm{OCOR}^{1}}^{\mathrm{Ar}}$

$(R)-1(\mathrm{a}-\mathrm{e})$

(S)-2(a-e)

\section{Scheme 2}

Table 4. Lipase PS-D-catalyzed kinetic resolution of $r a c-2(a-e)(0.1 \mathrm{M})$ with 1-butanol $(0.3 \mathrm{M})$ in TBME

\begin{tabular}{cccccccc}
\hline Entry & Compound & $\begin{array}{c}\text { Amount of lipase } \\
\left(\mathrm{mg} \mathrm{mL}^{-1}\right)\end{array}$ & $\begin{array}{c}\text { Time } \\
(\mathrm{h})\end{array}$ & $\begin{array}{c}\text { Conversion } \\
(\%)\end{array}$ & $\begin{array}{c}\mathrm{ee}^{(S)-2(\mathrm{a}-\mathrm{e})} \\
(\%)\end{array}$ & $\begin{array}{c}\mathrm{ee}^{(R)-1(\mathrm{a}-\mathrm{e})} \\
(\%)\end{array}$ & $E$ \\
\hline 1 & rac-2a & 50 & 2 & 48 & 93 & 98 & $» 200$ \\
2 & $r a c-2 b$ & 50 & 24 & 50 & 99 & 99 & $» 200$ \\
3 & $r a c-2 c$ & 50 & 1 & 50 & 98 & 98 & $» 200$ \\
4 & $r a c-2 d$ & 50 & 24 & 50 & 99 & 99 & $» 200$ \\
5 & $r a c-2 e$ & 50 & 120 & 25 & 33 & 99 & $» 200$ \\
6 & $r a c-2 e$ & 100 & 120 & 38 & 61 & 99 & $» 200$ \\
7 & $r a c-2 e^{\mathbf{a}}$ & 100 & 120 & 48 & 91 & 99 & $» 200$ \\
\hline
\end{tabular}

${ }^{\mathrm{a}} 47^{\circ} \mathrm{C}$

\section{Preparation of $(R)$ - and $(S)-1(a-e)$}

Finally the preparative scale resolution of $\mathrm{rac}-\mathbf{1}(\mathbf{a}-\mathbf{e})$ was performed under optimized conditions (Scheme 1). In accordance with excellent enantioselectivities, the reactions were stopped at 50\% conversion and the formed $R$-esters and unreacted $S$-alcohols were separated by column chromatography as described in the Experimental section. Compound rac-1f was not resolved as only moderate enantioselectivity was achieved (Table 3, entry 7). Lipase PS-D-catalyzed alcoholysis with 1-butanol in TBME afforded the corresponding $R$-alcohols. In general, both $(R)$ and $(S)$-1(a-e) were obtained in highly enantiopure forms as shown by the ee values and the consistency of the specific rotations for the antipodes (Table 5). The specific rotation +33.4 (entry 1 ) for the $(R)-\mathbf{1 a}(\mathrm{ee}=99 \%)$ is in accordance with the literature value ${ }^{1}-33.5$ calculated to the $S$-alcohol at $30^{\circ} \mathrm{C}$ under otherwise the same conditions. 
Table 5. Prepared enantiopure alcohols 1(a-e)

\begin{tabular}{ccccccc}
\hline Substrate & \multicolumn{3}{c}{$(R)$-alcohol } & \multicolumn{3}{c}{$(S)$-alcohol } \\
\cline { 2 - 7 } & ee $(\%)$ & ${\text { yield }(\%)^{\mathrm{a}, \mathrm{b}}}^{25}$ & {$[\alpha]_{D}^{25}$} & ee $(\%)$ & yield $(\%)^{\mathrm{b}}$ & {$[\alpha]_{D}^{25}$} \\
\hline 1a & 99 & 72 & +33.4 & 91 & 94 & -28.5 \\
1b & 99 & 90 & +13.1 & 99 & 85 & -13.7 \\
1c & 99 & 84 & +17.4 & 98 & 99 & -16.3 \\
1d & 99 & 77 & +35.4 & 98 & 98 & -33.9 \\
1e & 95 & 65 & +69.5 & 95 & 87 & -66.5 \\
\hline
\end{tabular}

${ }^{\mathrm{a} O v e r a l l ~ y i e l d s ~ t h e ~ t w o ~ e n z y m a t i c ~ s t e p s ; ~}$

${ }^{\mathrm{b}} 50 \%$ of the racemates taken as $100 \%$ theoretical yields.

\section{Conclusions}

Simple chemoenzymatic method for the preparation of the enantiomers of sulphur heteroaromatic $\beta$-hydroxy nitriles has been described starting from the corresponding aldehydes and using lipase PS-D catalysis as the source of enantiopurity.

\section{Experimental Section}

General Procedures. All solvents were of the highest analytical grade and were dried by standard methods when necessary. Aldehydes, (trimethylsilyl)acetonitrile, lithium acetate and vinyl acetate were purchased from Aldrich. Vinyl butanoate was from Fluka and isopropenyl acetate from Merck. 2,2,2-Trifluoroethyl butanoate was prepared from butanoyl chloride and the alcohol. Lipase PS from Burkholderia cepacia (previously Pseudomonas cepacia) immobilized on diatomaceous earth (lipase PS-D) and on ceramic particles (lipase PS-C II) and lipase AK-C from Pseudomonas fluorescence were purchased from Amano Europe, England. Candida antarctica lipase A (CAL-A) was the product of Roche, and before use it was adsorbed on celite as previously described. ${ }^{16}$ Candida antarctica lipase B (CAL-B, Novozym 435), Rhizomucur miehei lipase (Lipozyme RM IM) and Thermomyces lanuginosus lipase (Lipozyme TL IM) were obtained from Novozymes. The ${ }^{1} \mathrm{H}$ NMR and ${ }^{13} \mathrm{C}$ NMR spectra were recorded on a Brucker 500 MHz spectrometer operating at 500 and $125.77 \mathrm{MHz}$, respectively, with tetramethylsilane (TMS) as an internal standard. Mass spectra (MS) were taken on a VG 7070E mass spectrometer. IR spectra were recorded on Perkin Elmer Spectrum 1000 spectrometer. Analytical thin layer chromatography (TLC) was carried out on Merck Kieselgel $60 \mathrm{~F}_{254}$ sheets. Preparative chromatographic separations were performed using column chromatography on Merck Kieselgel $60(0.063-0.200 \mu \mathrm{m})$. Optical rotations $\left(c 1, \mathrm{CHCl}_{3}\right)$ were measured with a Perkin Elmer 341 Polarimeter and $[\alpha]_{D}^{25}$ values are given in units of $10^{-1} \mathrm{deg} \mathrm{cm}^{2} \mathrm{~g}^{-1}$. Melting points were recorded 
with Gallenkamp melting point apparatus and are uncorrected. The determination of $E$ was based on the equation $E=\ln \left[(1-c)\left(1-e_{\mathrm{S}}\right)\right] / \ln \left[(1-c)\left(1+\mathrm{ee}_{\mathrm{S}}\right)\right]$ with $c=\mathrm{ee}_{\mathrm{S}} /\left(\mathrm{ee}_{\mathrm{S}}+\mathrm{ee}_{\mathrm{P}}\right)$ using linear regression $\left\{E\right.$ as the slope of the line $\ln \left[(1-c)\left(1-e_{S}\right)\right]$ versus $\left.\ln \left[(1-c)\left(1+e_{S}\right)\right]\right\} .{ }^{17}$ The enzymatic reactions were performed at room temperature $\left(23{ }^{\circ} \mathrm{C}\right)$ if not otherwise stated.

\section{Preparation of rac-1(a-f)}

Racemates 1(a-f) were all prepared according to the known method. ${ }^{13}$ The preparation of rac-1c is used to illustrate the general procedure. (Trimethylsilyl)acetonitrile $(1.68 \mathrm{~g}, 14.86 \mathrm{mmol}$, $2.03 \mathrm{~mL})$ was added to the combined solution of lithium acetate $(10 \%$ molar $)$ in DMF $(18 \mathrm{~mL})$ and 5-phenylthiophene-2-carbaldehyde $(2.00 \mathrm{~g}, 10.62 \mathrm{mmol})$ in $\mathrm{DMF}(8 \mathrm{~mL})$ at $0{ }^{\circ} \mathrm{C}$. The mixture was allowed to warm slowy to room temperature, and the stirring was continued for $3 \mathrm{~h}$. The reaction was quenched with $\mathrm{HCl}(1 \mathrm{M}, 8 \mathrm{~mL})$ and $\mathrm{MeOH}(40 \mathrm{~mL})$. Distilled water $(60 \mathrm{~mL})$ was added and the mixture was extracted with diethyl ether $(3 \times 60 \mathrm{~mL})$. The organic layer was washed with brine, dried over $\mathrm{MgSO}_{4}$ and evaporated. The crude product was purified by passing through a silica gel column with ethyl acetate/hexane (3/7) as an eluent to afford 3hydroxy-3-(5-phenylthiophen-2-yl)propanenitrile, rac-1c (1.99 g, $8.67 \mathrm{mmol})$.

3-Hydroxy-3-(thiophen-2-yl)propanenitrile, rac-1a. Dark brown oil, yield 80\%; HRMS $\mathrm{M}^{+}$ found $\left(\mathrm{M}^{+}\right.$calculated for $\left.\mathrm{C}_{7} \mathrm{H}_{7} \mathrm{NOS}\right)$ : 153.02483 (153.02483); MS: $\mathrm{m} / \mathrm{z}$ (relative intensity)=153 (17), 136 (2), 113 (100), 110 (10), 85 (44), 58 (4), 45 (20); IR (neat, cm cm $^{-1} 3430$ (s), 3108 (m),

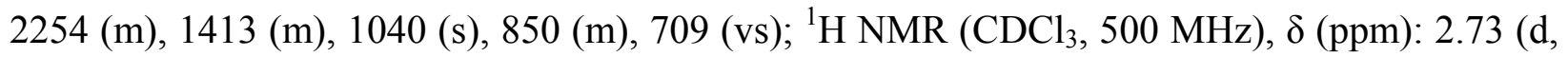
$J=4.3 \mathrm{~Hz}, 1 \mathrm{H}, \mathrm{OH}), 2.87\left(\mathrm{dd}, J=6.2 \mathrm{~Hz}, J=2.8 \mathrm{~Hz}, 2 \mathrm{H}, \mathrm{C}(2)-\mathrm{CH}(\mathrm{OH}) \underline{\mathrm{H}}_{2} \mathrm{CN}\right), 5.29$ (q, $J=5.5 \mathrm{~Hz}$, $\left.1 \mathrm{H}, \mathrm{C}(2)-\mathrm{CH}(\mathrm{OH}) \mathrm{CH}_{2} \mathrm{CN}\right), 7.01(\mathrm{dd}, J=5.0 \mathrm{~Hz}, J=3.5 \mathrm{~Hz}, 1 \mathrm{H}, \mathrm{C}(4)-\mathrm{H}), 7.09$ (d, $J=3.5 \mathrm{~Hz}, 1 \mathrm{H}$, $\mathrm{C}(3)-\mathrm{H}), 7.32$ (dd, $J=5.0 \mathrm{~Hz}, J=1.1 \mathrm{~Hz}, 1 \mathrm{H}, \mathrm{C}(5)-\mathrm{H}) ;{ }^{13} \mathrm{C} \mathrm{NMR}\left(\mathrm{CDCl}_{3}, 126 \mathrm{MHz}\right), \delta(\mathrm{ppm})$ : $28.21\left(\mathrm{C}(2)-\mathrm{CH}(\mathrm{OH}) \underline{\left.\mathrm{CH}_{2} \mathrm{CN}\right),} 66.32\left(\mathrm{C}(2)-\underline{\mathrm{CH}}(\mathrm{OH}) \mathrm{CH}_{2} \mathrm{CN}\right), 116.88(\mathrm{CN}), 124.78(\mathrm{C}(3))\right.$, $125.83(\mathrm{C}(5)), 127.12(\mathrm{C}(4)), 144.37(\mathrm{C}(2))$.

3-(5-Bromothiophen-2-yl)-3-hydroxypropanenitrile, rac-1b. Dark brown oil, yield 91\%; HRMS $\mathrm{M}^{+}$found $\left(\mathrm{M}^{+}\right.$calculated for $\mathrm{C}_{7} \mathrm{H}_{6} \mathrm{BrNOS}$ ): 230.93450 (230.93534); $\mathrm{MS}: \mathrm{m} / z$ (relative intensity) = 233 (19), 231 (19), 193 (91), 191 (100), 84 (65); IR (neat, cm ${ }^{-1}$ ): 3427 (vs); 3100

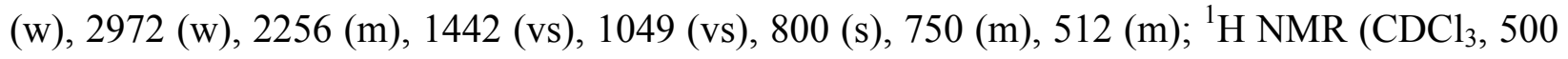
$\mathrm{MHz}), \delta(\mathrm{ppm}): 2.74(\mathrm{~d}, J=4.4 \mathrm{~Hz}, 1 \mathrm{H}, \mathrm{OH}), 2.83(\mathrm{dd}, J=6.2 \mathrm{~Hz}, J=1.4 \mathrm{~Hz}, 2 \mathrm{H}, \mathrm{C}(2)-$ $\left.\mathrm{CH}(\mathrm{OH}) \underline{\mathrm{H}}_{2} \mathrm{CN}\right), 5.19$ (q, $\left.J=5.4 \mathrm{~Hz}, 1 \mathrm{H}, \mathrm{C}(2)-\mathrm{CH}(\mathrm{OH}) \mathrm{CH}_{2} \mathrm{CN}\right), 6.82$ (dd, $J=3.7 \mathrm{~Hz}, J=0.6 \mathrm{~Hz}$, $1 \mathrm{H}, \mathrm{C}(3)-\mathrm{H}), 6.95$ (d, J=3.8 Hz, $1 \mathrm{H}, \mathrm{C}(4)-\mathrm{H}) ;{ }^{13} \mathrm{C} \mathrm{NMR}\left(\mathrm{CDCl}_{3}, 126 \mathrm{MHz}\right), \delta(\mathrm{ppm}): 28.04$ $\left(\mathrm{C}(2)-\mathrm{CH}(\mathrm{OH}) \underline{\mathrm{CH}}_{2} \mathrm{CN}\right), 66.34\left(\mathrm{C}(2)-\underline{\mathrm{CH}}(\mathrm{OH}) \mathrm{CH}_{2} \mathrm{CN}\right), 112.88(\mathrm{C}(5)) 116.64(\mathrm{CN}), 125.01$ (C(3)), 129.82 (C(4)), 145.99 (C(2)).

3-Hydroxy-3-(5-phenylthiophen-2-yl)propanenitrile, rac-1c. Yellow solid (m.p. 92-93 $\left.{ }^{\circ} \mathrm{C}\right)$, yield 82\%; HRMS $\mathrm{M}^{+}$found ( $\mathrm{M}^{+}$calculated for $\mathrm{C}_{13} \mathrm{H}_{11} \mathrm{NOS}$ ): 229.05570 (229.05613); MS: $\mathrm{m} / z$ (relative intensity) = $229(20), 190$ (13), 189 (100), 161 (11), 128 (22), 115 (15), 77 (5); IR (KBr, $\mathrm{cm}^{-1}$ ): 3429 (vs), 3017 (w), 2889 (w), 2260 (m), 1467 (m), 1062 (s), 804 (s), 756 (vs), 687 (s); ${ }^{1} \mathrm{H}$ NMR $\left(\mathrm{CDCl}_{3}, 500 \mathrm{MHz}\right), \delta(\mathrm{ppm}): 2.70(\mathrm{~d}, J=4.2 \mathrm{~Hz}, 1 \mathrm{H}, \mathrm{OH}), 2.89$ (dd, $J=6.2 \mathrm{~Hz}, J=3.1 \mathrm{~Hz}$, $\left.2 \mathrm{H}, \mathrm{C}(2)-\mathrm{CH}(\mathrm{OH}) \underline{\mathrm{H}}_{2} \mathrm{CN}\right), 5.27$ (q, $\left.J=5.5 \mathrm{~Hz}, 1 \mathrm{H}, \mathrm{C}(2)-\mathrm{C} \underline{\mathrm{H}}(\mathrm{OH}) \mathrm{CH}_{2} \mathrm{CN}\right), 7.05$ (dd, $J=3.7 \mathrm{~Hz}$, 
$J=0.7 \mathrm{~Hz}, 1 \mathrm{H}, \mathrm{C}(3)-\mathrm{H}), 7.18(\mathrm{~d}, J=3.7 \mathrm{~Hz}, 1 \mathrm{H}, \mathrm{C}(4)-\mathrm{H}), 7.30$ (t, $\left.J=7.4 \mathrm{~Hz}, 1 \mathrm{H}, \mathrm{C}\left(4^{\prime}\right)-\mathrm{H}\right), 7.37$ (t, $\left.J=7.4 \mathrm{~Hz}, 2 \mathrm{H}, \mathrm{C}\left(3^{\prime}\right)-\mathrm{H}, \mathrm{C}\left(5^{\prime}\right)-\mathrm{H}\right), 7.56$ (dd, J=8.1 Hz, J=1.0 Hz, 2H, C(2')-H, C(6')-H); ${ }^{13} \mathrm{C}$

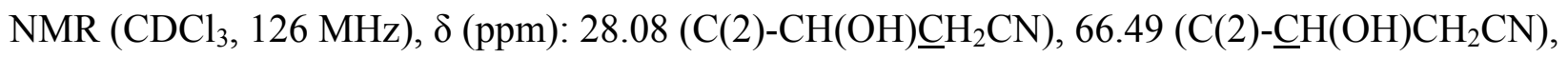
116.85 (CN), 122.84 (C(3)), 125.73 (C(4)), 125.85 (C(2’), C(6’)), 127.94 (C(4')), 128.98 (C(3’), $\left.\mathrm{C}\left(5^{\prime}\right)\right), 133.82$ (C(1'), 143.47 (C(5)), 144.95 (C(2)).

3-(Benzo $[b]$ thiophen-2-yl)-3-hydroxypropanenitrile, rac-1d. Dark yellow solid (m.p. 112$113{ }^{\circ} \mathrm{C}$ ), yield 65\%; HRMS $\mathrm{M}^{+}$found $\left(\mathrm{M}^{+}\right.$calculated for $\mathrm{C}_{11} \mathrm{H}_{9} \mathrm{NOS}$ ): 203.04120 (203.04048); MS: $m / z$ (relative intensity) = 203 (41), 191 (9), 164 (11), 163 (100), 135 (41), 134 (18), 91 (28), 89 (12); IR (KBr, cm ${ }^{-1}$ ): 3420 (vs), 2929 (w), 2261 (s), 1457 (m), 1064 (s), 831 (s), 751 (vs), 729 (s); ${ }^{1} \mathrm{H} \mathrm{NMR}\left(\mathrm{CDCl}_{3}, 500 \mathrm{MHz}\right), \delta(\mathrm{ppm}): 2.76(\mathrm{~d}, J=4.4,1 \mathrm{H}, \mathrm{OH}), 2.93(\mathrm{dd}, J=6.2 \mathrm{~Hz}, J=2.5$ $\left.\mathrm{Hz}, 2 \mathrm{H}, \mathrm{C}(2)-\mathrm{CH}(\mathrm{OH}) \underline{\mathrm{H}}_{2} \mathrm{CN}\right), 5.35$ (q, J=5.4 Hz, 1H, C(2)- $\left.\underline{\mathrm{H}}(\mathrm{OH}) \mathrm{CH}_{2} \mathrm{CN}\right), 7.31$ (s, 1H, C(3)$\mathrm{H})$, 7.33-7.39 (m, 2H, C(5)-H, C(6)-H), 7.73-7.77 (m, 1H, C(4)-H), 7.81-7.84 (m, 1H, C(7)-H); ${ }^{13} \mathrm{C} \quad \mathrm{NMR}\left(\mathrm{CDCl}_{3}, 126 \mathrm{MHz}\right), \delta(\mathrm{ppm}): 27.95 \quad\left(\mathrm{C}(2)-\mathrm{CH}(\mathrm{OH}) \underline{\mathrm{CH}}_{2} \mathrm{CN}\right), 66.92 \quad(\mathrm{C}(2)-$ $\left.\underline{\mathrm{CH}}(\mathrm{OH}) \mathrm{CH}_{2} \mathrm{CN}\right), 116.66(\mathrm{CN}), 121.48(\mathrm{C}(3)), 122.57$ (C(7)), $123.98(\mathrm{C}(4)), 124.76(\mathrm{C}(5))$, 124.99 (C(6)), 139.11 (C(8)), 139.30 (C(9)), 144.70 (C(2)).

3-(Benzo[b]thiophen-3-yl)-3-hydroxypropanenitrile, rac-1e. Light brown oil, yield 87\%; HRMS $\mathrm{M}^{+}$found $\left(\mathrm{M}^{+}\right.$calculated for $\left.\mathrm{C}_{11} \mathrm{H}_{9} \mathrm{NOS}\right)$ : 203.03990 (203.04048); MS: $\mathrm{m} / z$ (relative intensity) = 203 (44), 191 (10), 163 (100), 135 (48), 91 (28); IR (neat, cm ${ }^{-1}$ ): 3437 (s), 2253 (m), 1428 (s), 1067 (m), 763 (s), 735 (vs); ${ }^{1} \mathrm{H}$ NMR $\left(\mathrm{CDCl}_{3}, 500 \mathrm{MHz}\right), \delta$ (ppm): 2.95 (dd, $J=12.5$ $\left.\mathrm{Hz}, J=6.0 \mathrm{~Hz}, 2 \mathrm{H}, \mathrm{C}(3)-\mathrm{CH}(\mathrm{OH}) \underline{\mathrm{H}}_{2} \mathrm{CN}\right), 5.39$ (q, $\left.J=3.9 \mathrm{~Hz}, 1 \mathrm{H}, \mathrm{C}(3)-\mathrm{CH}(\mathrm{OH}) \mathrm{CH}_{2} \mathrm{CN}\right)$, 7.367.42 (m, 2H, C(5)-H, C(6)-H), 7.52 (s, 1H, C(2)-H), 7.78-7.80 (m, 1H, C(4)-H), 7.86-7.88 (m, $1 \mathrm{H}, \mathrm{C}(7)-\mathrm{H}) ;{ }^{13} \mathrm{C} \mathrm{NMR}\left(\mathrm{CDCl}_{3}, 126 \mathrm{MHz}\right), \delta(\mathrm{ppm}): 26.46\left(\mathrm{C}(3)-\mathrm{CH}(\mathrm{OH}) \mathrm{CH}_{2} \mathrm{CN}\right), 65.71(\mathrm{C}(3)-$ $\left.\underline{\mathrm{CH}}(\mathrm{OH}) \mathrm{CH}_{2} \mathrm{CN}\right), 117.31(\mathrm{CN}), 121.58(\mathrm{C}(2)), 123.21(\mathrm{C}(7)), 123.88(\mathrm{C}(4)), 124.52(\mathrm{C}(5))$, 124.91 (C(6)), 135.89 (C(8)), 136.25 (C(9)), 140.98 (C(3)).

3-Hydroxy-3-(thiazol-2-yl)propanenitrile, rac-1f. Yellow solid (m.p. 79-80 ${ }^{\circ} \mathrm{C}$ ), yield 41\%; HRMS $\mathrm{M}^{+}$found $\left(\mathrm{M}^{+}\right.$calculated for $\left.\mathrm{C}_{6} \mathrm{H}_{6} \mathrm{~N}_{2} \mathrm{OS}\right)$ : 154.01990 (154.02008); $\mathrm{MS}: \mathrm{m} / z$ (relative intensity) = 154 (15), 114 (100), 86 (25), 58 (27); IR (KBr, cm $\left.{ }^{-1}\right): 3327(\mathrm{~s}), 2905$ (w), 2249 (m), 1501 (s), 1413 (m), 1084 (vs), 761 (s), 615 (s); ${ }^{1} \mathrm{H}$ NMR (CDCl, $\left.500 \mathrm{MHz}\right), \delta$ (ppm): 2.96 (dd, $J=16.7 \mathrm{~Hz}, J=7.2 \mathrm{~Hz}, 1 \mathrm{H}, \mathrm{C}(2)-\mathrm{CH}(\mathrm{OH}) \underline{\mathrm{H}}_{2} \mathrm{CN}$ ), 3.13 (dd, $J=16.7 \mathrm{~Hz}, J=4.4 \mathrm{~Hz}, 1 \mathrm{H}, \mathrm{C}(2)-$ $\left.\mathrm{CH}(\mathrm{OH}) \mathrm{CNC}_{2} \mathrm{CN}\right), 4.08$ (d, J=3.7 Hz, 1H, OH), 5.34 (t, J=3.3 Hz, 1H, C(2)-C $\left.\underline{\mathrm{H}}(\mathrm{OH}) \mathrm{CH}_{2} \mathrm{CN}\right)$, 7.39 (d, $J=3.2 \mathrm{~Hz}, 1 \mathrm{H}, \mathrm{C}(4)-\mathrm{H}), 7.78$ (d, $J=3.2 \mathrm{~Hz}, 1 \mathrm{H}, \mathrm{C}(5)-\mathrm{H}) ;{ }^{13} \mathrm{C} \mathrm{NMR}\left(\mathrm{CDCl}_{3}, 126 \mathrm{MHz}\right), \delta$ (ppm): 26.90 (C(2)-CH(OH) $\left.\underline{\mathrm{CH}}_{2} \mathrm{CN}\right), 67.74\left(\mathrm{C}(2)-\underline{\mathrm{CH}}(\mathrm{OH}) \mathrm{CH}_{2} \mathrm{CN}\right), 116.72(\mathrm{CN}), 120.35$ (C(4)), $142.80(\mathrm{C}(5)), 170.79$ (C(2)).

\section{Lipase-catalyzed acylation of racemic $\beta$-hydroxynitriles 1(a-f)}

In a typical small-scale experiment, lipase was added to the solution of $\mathrm{rac}-\mathbf{1}(\mathbf{a}-\mathbf{f})(0.1 \mathrm{M})$ and an acyl donor $(0.2 \mathrm{M})$ in an organic solvent. The reaction mixture was shaken at room temperature. The progress of the reaction and the ee values of the products were followed by taking samples $(10 \mu \mathrm{L})$ at intervals and analyzing them by HP 1090 Liquid Chromatograph equipped with Daicel Chiralcel $(0.46$ X $25 \mathrm{~cm})$ OD-H column for compounds 1(b-f). In the case of compound 
1a the samples $(100 \mu \mathrm{L})$ were analyzed with a HP 6850 Series GC System equipped with a Cyclosil-B (30 m X 0.32 mm X $0.25 \mu \mathrm{m})$ column.

\section{Lipase-catalyzed preparative scale kinetic resolution of rac-1(a-e)}

The general procedure is described for $r a c-1 c$ as a model compound. Lipase PS-D (1.09 g) was added to the solution of rac-1c $(1.00 \mathrm{~g}, 4.36 \mathrm{mmol})$ and vinyl acetate $(0.75 \mathrm{~g}, 8.72 \mathrm{mmol}$, $0.80 \mathrm{~mL})$ in TBME $(43 \mathrm{~mL})$, and the mixture was shaken at room temperature for $1 \mathrm{~h}$. The reaction was stopped by filtering off the enzyme. The enzyme was washed twice with TBME $(2 \times 10 \mathrm{ml})$. After evaporation, the unreacted $(S)$-1c and the produced $(R)$-2c were separated on silica gel by elution with ethyl acetate/hexane (3/7).

In the case of rac-1e vinyl butanoate was used as an acyl donor due to analytical problems in achieving good enantioseparation for the acetate product with the chiral HPLC method.

(S)-3-Hydroxy-3-(thiophen-2-yl)propanenitrile, $(\boldsymbol{S})$-1a. Brown oil, yield 94\%; $[\alpha]_{D}^{25}=-28.5(c$ $\left.1, \mathrm{CHCl}_{3}\right)$, ee $=91 \%$.

(R)-2-Cyano-1-(thiophen-2-yl)ethyl acetate, $(\boldsymbol{R})-2 \mathbf{a}$. Light brown oil, yield 88\%; $[\alpha]_{D}^{25}=+94.0$ (c 1, $\mathrm{CHCl}_{3}$ ), ee=95\%; HRMS $\mathrm{M}^{+}$found $\left(\mathrm{M}^{+}\right.$calculated for $\left.\mathrm{C}_{9} \mathrm{H}_{9} \mathrm{NO}_{2} \mathrm{~S}\right)$ : 195.03550 (195.03540); MS: $m / z$ (relative intensity) = 195 (37), 153 (25), 136 (35), 113 (100), 85 (10), 43 (63); IR (neat, $\mathrm{cm}^{-1}$ ): 3100 (m), 2910 (m), 2252 (m), 1730 (s), 1300 (s), 1200 (vs), 850 (s), 709 (vs); ${ }^{1} \mathrm{H} \mathrm{NMR}\left(\mathrm{CDCl}_{3}, 500 \mathrm{MHz}\right), \delta(\mathrm{ppm}): 2.14$ (s, 3H, OCOCH 3$), 2.99$ (dd, $J=6.0 \mathrm{~Hz}, J=2.1$ $\left.\mathrm{Hz}, 2 \mathrm{H}, \mathrm{CH}\left(\mathrm{OCOCH}_{3}\right) \underline{\mathrm{H}}_{2} \mathrm{CN}\right), 6.27\left(\mathrm{t}, J=6.2 \mathrm{~Hz}, 1 \mathrm{H}, \mathrm{CH}\left(\mathrm{OCOCH}_{3}\right) \mathrm{CH}_{2} \mathrm{CN}\right), 7.01$ (dd, $J=5.1$ $\mathrm{Hz}, J=3.6 \mathrm{~Hz}, 1 \mathrm{H}, \mathrm{C}(4)-\mathrm{H}), 7.16$ (d, $J=3.5 \mathrm{~Hz}, 1 \mathrm{H}, \mathrm{C}(3)-\mathrm{H}), 7.34$ (dd, $J=5.1 \mathrm{~Hz}, J=1.0 \mathrm{~Hz}, 1 \mathrm{H}$, $\mathrm{C}(5)-\mathrm{H}) ; \quad{ }^{13} \mathrm{C} \quad \mathrm{NMR} \quad\left(\mathrm{CDCl}_{3}, \quad 500 \quad \mathrm{MHz}\right), \quad \delta \quad(\mathrm{ppm}): \quad 20.86 \quad\left(\mathrm{OCO}_{\underline{H}}\right), \quad 25.64$ $\left(\mathrm{CH}\left(\mathrm{OCOCH}_{3}\right) \underline{\left.\mathrm{CH}_{2} \mathrm{CN}\right),} 66.27\left(\underline{\mathrm{CH}}\left(\mathrm{OCOCH}_{3}\right) \mathrm{CH}_{2} \mathrm{CN}\right), 115.76(\mathrm{CN}), 126.49(\mathrm{C}(3)), 126.62\right.$ $(\mathrm{C}(5)), 127.06(\mathrm{C}(4)), 139.37(\mathrm{C}(2)), 169.50\left(\mathrm{OCOCH}_{3}\right)$.

(S)-3-(5-Bromothiophen-2-yl)-3-hydroxypropanenitrile, $(\boldsymbol{S})-1 \mathrm{~b}$. Dark brown oil, yield 85\%; $[\alpha]_{D}^{25}=-13.7\left(c 1, \mathrm{CHCl}_{3}\right)$, ee $=99 \%$.

(R)-1-(5-Bromothiophen-2-yl)-2-cyanoethylacetate, $(\boldsymbol{R})$-2b. Brown oil, yield 92\%; $[\alpha]_{D}^{25}=$ +96.4 ( c 1, $\left.\mathrm{CHCl}_{3}\right)$, ee>99 \%; HRMS M${ }^{+}$found $\left(\mathrm{M}^{+}\right.$calculated for $\left.\mathrm{C}_{9} \mathrm{H}_{8} \mathrm{BrNO}_{2} \mathrm{~S}\right): 272.94540$ (272.94591); MS: $m / z$ (relative intensity) = 273 (22), 248 (5), 233 (16), 191 (95), 135 (23), 43 (100); IR (neat, $\mathrm{cm}^{-1}$ ): 2975 (w), 2254 (w), 1751 (vs), 1441 (m), 1371 (m), 1222 (vs), 1038 (m), $802(\mathrm{~m}), 600(\mathrm{w}) ;{ }^{1} \mathrm{H} \mathrm{NMR}\left(\mathrm{CDCl}_{3}, 500 \mathrm{MHz}\right), \delta(\mathrm{ppm}): 2.14$ (s, 3H, OCOC$\left.\underline{H}_{3}\right), 2.96(\mathrm{~d}, J=6.2$ $\left.\mathrm{Hz}, 2 \mathrm{H}, \mathrm{CH}\left(\mathrm{OCOCH}_{3}\right) \mathrm{CH}_{2} \mathrm{CN}\right), 6.14\left(\mathrm{t}, J=6.4 \mathrm{~Hz}, 1 \mathrm{H},-\mathrm{CH}\left(\mathrm{OCOCH}_{3}\right) \mathrm{CH}_{2} \mathrm{CN}\right), 6.92(\mathrm{dd}, J=4.0$ $\mathrm{Hz}, J=0.4 \mathrm{~Hz}, 1 \mathrm{H}, \mathrm{C}(3)-\mathrm{H}), 6.96(\mathrm{~d}, J=3.8 \mathrm{~Hz}, 1 \mathrm{H}, \mathrm{C}(4)-\mathrm{H}) ;{ }^{13} \mathrm{C} \mathrm{NMR}\left(\mathrm{CDCl}_{3}, 126 \mathrm{MHz}\right), \delta$

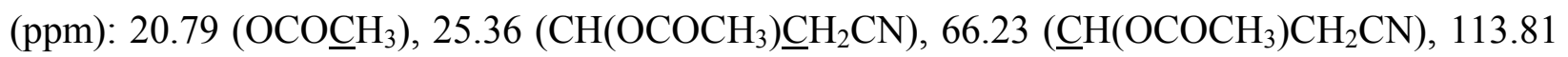
$(\mathrm{C}(5)), 115.43(\mathrm{CN}), 127.05(\mathrm{C}(3)), 129.79$ (C(4)), 140.80 (C(2)), $169.36\left(\mathrm{OCOCH}_{3}\right)$.

(S)-3-Hydroxy-3-(5-phenylthiophen-2-yl)propanenitrile, (S)-1c. Yellow solid (m.p. 95$\left.96{ }^{\circ} \mathrm{C}\right)$, yield $99 \% ;[\alpha]_{D}^{25}=-16.3\left(c 1, \mathrm{CHCl}_{3}\right)$, ee $=98 \%$.

(R)-2-Cyano-1-(5-phenylthiophen-2-yl)ethyl acetate, $(\boldsymbol{R})-2 \mathrm{c}$. Yellow solid (m.p. 83-85 $\left.{ }^{\circ} \mathrm{C}\right)$, yield $97 \%$; $\left.[\alpha]_{D}^{25}=+138.2(c) 1, \mathrm{CHCl}_{3}\right)$, ee=98\%; HRMS $\mathrm{M}^{+}$found $\left(\mathrm{M}^{+}\right.$calculated for 
$\mathrm{C}_{15} \mathrm{H}_{13} \mathrm{NO}_{2} \mathrm{~S}$ ): 271.06620 (271.06670); MS: m/z (relative intensity) = 271 (31), 211 (24), 189 (100), 115 (14), 77 (3), 43 (15); IR (KBr, cm $\left.{ }^{-1}\right): 2254$ (m), 1743 (s), 1445 (m), 1377 (m), 1231 (vs), 1027 (s), 816 (m), 759 (s), $688(\mathrm{~m}) ;{ }^{1} \mathrm{H} \mathrm{NMR}\left(\mathrm{CDCl}_{3}, 500 \mathrm{MHz}\right.$ ), $\delta$ (ppm): 2.15 (s, 3H, $\left.\mathrm{OCOC}_{3}\right)_{3}, 3.02\left(\mathrm{dd}, J=6.5 \mathrm{~Hz}, J=2.1 \mathrm{~Hz}, 2 \mathrm{H}, \mathrm{CH}\left(\mathrm{OCOCH}_{3}\right) \underline{\mathrm{C}}_{2} \mathrm{CN}\right), 6.24$ (t, $J=6.4 \mathrm{~Hz}$, $\left.\mathrm{C} \underline{\mathrm{H}}\left(\mathrm{OCOCH}_{3}\right) \mathrm{CH}_{2} \mathrm{CN}\right), 7.12(\mathrm{dd}, J=3.5 \mathrm{~Hz}, J=0.6 \mathrm{~Hz}, 1 \mathrm{H}, \mathrm{C}(3)-\mathrm{H}), 7.18$ (d, $J=3.7 \mathrm{~Hz}, 1 \mathrm{H}, \mathrm{C}(4)-$ H), 7.30 (t, $\left.J=7.4 \mathrm{~Hz}, 1 \mathrm{H}, \mathrm{C}\left(4^{\prime}\right)-\mathrm{H}\right), 7.38$ (t, $\left.J=7.3 \mathrm{~Hz}, 2 \mathrm{H}, \mathrm{C}\left(3^{\prime}\right)-\mathrm{H}, \mathrm{C}\left(5^{\prime}\right)-\mathrm{H}\right), 7.57$ (dd, $J=8.3$ $\left.\mathrm{Hz}, J=1.2 \mathrm{~Hz}, 2 \mathrm{H}, \mathrm{C}\left(2^{\prime}\right)-\mathrm{H}, \mathrm{C}\left(6^{\prime}\right)-\mathrm{H}\right) ;{ }^{13} \mathrm{C} \mathrm{NMR}\left(\mathrm{CDCl}_{3}, 126 \mathrm{MHz}\right), \delta(\mathrm{ppm}): 20.89\left(\mathrm{OCOCH}_{3}\right)$, $25.47\left(\mathrm{O}\left(\mathrm{COCH}_{3}\right) \underline{\mathrm{CH}_{2} \mathrm{CN}}\right), 66.47\left(\underline{\mathrm{CH}}\left(\mathrm{OCOCH}_{3}\right) \mathrm{CH}_{2} \mathrm{CN}\right), 115.74(\mathrm{CN}), 122.79(\mathrm{C}(3)), 125.95$ (C(2’), C(6’)), 127.64 (C(4)), 128.09 (C(4')), 128.99 (C(3'), C(5')), 133.64 (C(1')), 138.38 $(\mathrm{C}(5)), 145.73(\mathrm{C}(2)), 169.54\left(\mathrm{OCOCH}_{3}\right)$.

(S)-3-(Benzo[b]thiophen-2-yl)-3-hydroxypropanenitrile, (S)-1d. Yellow solid (m.p. 106$\left.109{ }^{\circ} \mathrm{C}\right)$, yield 99\%; $[\alpha]_{D}^{25}=-33.9\left(c 1, \mathrm{CHCl}_{3}\right)$, ee $=98 \%$.

(R)-1-(Benzo[b]thiophen-2-yl)-2-cyanoethyl acetate, (R)-2d. Yellow solid (m.p. 90-91 ${ }^{\circ} \mathrm{C}$ ), yield $84 \% ;[\alpha]_{D}^{25}=+106.5\left(c 1, \mathrm{CHCl}_{3}\right)$, ee $>99 \%$; HRMS $\mathrm{M}^{+}$found $\left(\mathrm{M}^{+}\right.$calculated for $\mathrm{C}_{13} \mathrm{H}_{11} \mathrm{NO}_{2} \mathrm{~S}$ ): 245.05160 (245.05105); MS: $m / z$ (relative intensity) = 245 (42), 203 (22), 186 (22), 163 (100), 135 (13), 89 (10), 43 (30); IR (KBr, cm $\left.{ }^{-1}\right): 2941$ (s), 2250 (m), 1751 (vs), 1458

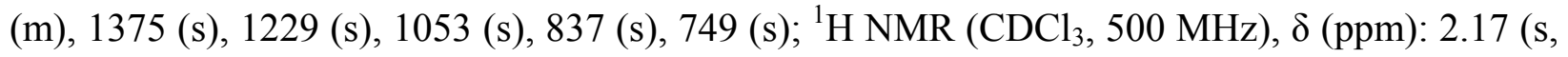
$\left.3 \mathrm{H}, \mathrm{OCOCH}_{3}\right), 3.05\left(\mathrm{dd}, J=6.0 \mathrm{~Hz}, J=2.1 \mathrm{~Hz}, 2 \mathrm{H}, \mathrm{CH}\left(\mathrm{OCOCH}_{3}\right) \underline{\mathrm{H}}_{2} \mathrm{CN}\right), 6.32(\mathrm{t}, J=6.0 \mathrm{~Hz}, 1 \mathrm{H}$, $\left.\mathrm{C} \underline{\mathrm{H}}\left(\mathrm{OCOCH}_{3}\right) \mathrm{CH}_{2} \mathrm{CN}\right)$, 7.34-7.38 (m, 2H, C(5)-H, C(6)-H), 7.39 (s, 1H, C(3)-H), 7.76-7.78 (m, $1 \mathrm{H}, \mathrm{C}(4)-\mathrm{H}), 7.81-7.82(\mathrm{~m}, 1 \mathrm{H}, \mathrm{C}(7)-\mathrm{H}) ;{ }^{13} \mathrm{C} \mathrm{NMR}\left(\mathrm{CDCl}_{3}, 126 \mathrm{MHz}\right), \delta(\mathrm{ppm}): 20.82$ $\left(\mathrm{OCO}^{-} H_{3}\right), 25.44\left(\mathrm{CH}\left(\mathrm{OCOCH}_{3}\right) \underline{\mathrm{CH}}_{2} \mathrm{CN}\right), 66.90\left(\underline{\mathrm{CH}}\left(\mathrm{OCOCH}_{3}\right) \mathrm{CH}_{2} \mathrm{CN}\right), 115.58(\mathrm{CN}), 122.47$ (C(3)), 123.38 (C(7)), 124.20 (C(4)), 124.83 (C(5)), 125.26 (C(6)), 138.82 (C(8)), 139.42 (C(9)), $139.77(\mathrm{C}(2)), 169.42\left(\mathrm{OCOCH}_{3}\right)$.

(S)-3-(Benzo[b]thiophen-3-yl)-3-hydroxypropanenitrile, $(\boldsymbol{S})$-1e. Brown oil, yield 87\%; $[\alpha]_{D}^{25}=$ $-66.5\left(\right.$ c $\left.1, \mathrm{CHCl}_{3}\right)$, ee=95\%.

(R)-1-(Benzo[b]thiophen-3-yl)-2-cyanoethyl butyrate, $(\boldsymbol{R})$-2e. Yellow oil, yield $78 \% ;[\alpha]_{D}^{25}=$ +53.6 ( c 1, $\left.\mathrm{CHCl}_{3}\right)$, ee=98\%; HRMS M $\mathrm{M}^{+}$found $\left(\mathrm{M}^{+}\right.$calculated for $\left.\mathrm{C}_{15} \mathrm{H}_{15} \mathrm{NO}_{2} \mathrm{~S}\right): 273.08310$ (273.08235); MS: $m / z$ (relative intensity) = 273 (49), 233 (8), 203 (21), 186 (37), 163 (79), 71 (100), 43 (45); IR (neat, cm ${ }^{-1}$ ): 3084 (w), 2875 (m), 2252 (m), 1741 (vs), 1526 (w), 1461 (m),

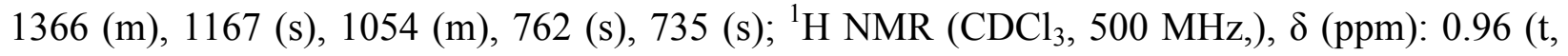
$J=7.4 \mathrm{~Hz}, 3 \mathrm{H}, \mathrm{OCOCH}_{2} \mathrm{CH}_{2} \underline{\mathrm{H}}_{3}$ ), 1.70 (sextet, $\left.J=7.4 \mathrm{~Hz}, 2 \mathrm{H}, \mathrm{OCOCH}_{2} \underline{\mathrm{H}}_{2} \mathrm{CH}_{3}\right), 2.42(\mathrm{~m}, 2 \mathrm{H}$, $\left.\mathrm{OCOC}_{2} \mathrm{CH}_{2} \mathrm{CH}_{3}\right), 3.07$ (dd, $\left.J=10.1 \mathrm{~Hz}, J=5.4 \mathrm{~Hz}, 2 \mathrm{H}, \mathrm{CH}\left(\mathrm{OCOCH}_{2} \mathrm{CH}_{2} \mathrm{CH}_{3}\right) \mathrm{C}_{2} \mathrm{CN}\right), 6.43(\mathrm{t}$, $\left.J=6.2 \mathrm{~Hz}, 1 \mathrm{H}, \mathrm{CH}\left(\mathrm{OCOCH}_{2} \mathrm{CH}_{2} \mathrm{CH}_{3}\right) \mathrm{CH}_{2} \mathrm{CN}\right), 7.39-7.44$ (m, 2H, C(5)-H, C(6)-H), 7.60 (s, 1H, $\mathrm{C}(2)-\mathrm{H}), 7.77-7.81(\mathrm{~m}, 1 \mathrm{H}, \mathrm{C}(4)-\mathrm{H}), 7.86-7.89(\mathrm{~m}, 1 \mathrm{H}, \mathrm{C}(7)-\mathrm{H}) ;{ }^{13} \mathrm{C} \mathrm{NMR}\left(\mathrm{CDCl}_{3}, 126 \mathrm{MHz}\right), \delta$ (ppm): 13.61 $\left(\mathrm{OCOCH}_{2} \mathrm{CH}_{2} \mathrm{CH}_{3}\right), 18.36\left(\mathrm{OCOCH}_{2} \mathrm{CH}_{2} \mathrm{CH}_{3}\right), 24.29\left(\mathrm{CH}\left(\mathrm{OCOCH}_{2} \mathrm{CH}_{2} \mathrm{CH}_{2} \mathrm{CH}_{3}\right) \underline{\mathrm{CH}}_{2}\right.$ $\mathrm{CN}), \quad 36.03\left(\mathrm{OCOCH}_{2} \mathrm{CH}_{2} \mathrm{CH}_{3}\right), \quad 65.60 \quad\left(\underline{\mathrm{CH}}\left(\mathrm{OCOCH}_{2} \mathrm{CH}_{2} \mathrm{CH}_{2} \mathrm{CH}_{3}\right) \mathrm{CH}_{2} \mathrm{CN}\right), 115.94$ (CN), 121.36 (C(2)), 123.24 (C(7)), 124.67 (C(5)), 124.99 (C(6)), 125.29 (C(4)), 131.86 (C(8)), 136.10 $(\mathrm{C}(9)), 140.74(\mathrm{C}(3)), 172.32\left(\mathrm{OCOCH}_{2} \mathrm{CH}_{2} \mathrm{CH}_{3}\right)$. 


\section{Lipase-catalyzed deprotection of $(R)-2(a-e)$ by alcoholysis}

The general procedure is described for rac-2c as a model compound. Lipase PS-D $(0.27 \mathrm{~g})$ and 1-butanol $(0.15 \mathrm{~mL})$ were added to the solution of $(R)-2 \mathrm{c}(0.15 \mathrm{~g}, 0.55 \mathrm{mmol})$ in TBME $(5.50 \mathrm{~mL})$, and the mixture was shaken at room temperature for $1 \mathrm{~h}$ (to reach the conversion higher than 95\%). The reaction was stopped by filtering off the enzyme. The produced $(R)-1 \mathrm{c}$ was purified on a short silica gel column by elution with ethyl acetate/hexane (3/7).

Conversion for the alcoholysis of $(R)$-1a was obtained using dihexyl ether as an external standard and for $(R)$-1b using acetophenone as an external standard. 1,4-dimethoxybenzene was used in all the other cases.

(R)-3-Hydroxy-3-(thiophen-2-yl)propanenitrile, $(\boldsymbol{R})$-1a. Light yellow oil, yield 85\%; $[\alpha]_{D}^{25}=$ $+33.4\left(c 1, \mathrm{CHCl}_{3}\right)$, ee $=99 \%$.

(R)-3-(5-Bromothiophen-2-yl)-3-hydroxypropanenitrile, $(\boldsymbol{R})-1 \mathbf{b}$. Yellow oil, yield 98\%; $[\alpha]_{D}^{25}$ $=+13.1\left(c 1, \mathrm{CHCl}_{3}\right)$, ee $=99 \%$.

(R)-3-Hydroxy-3-(5-phenylthiophen-2-yl)propanenitrile, (R)-1c. Yellow solid (m.p. 99$\left.101{ }^{\circ} \mathrm{C}\right)$, yield $87 \% ;[\alpha]_{D}^{25}=+17.4\left(c 1, \mathrm{CHCl}_{3}\right)$, ee $=99 \%$

(R)-3-(Benzo[b]thiophen-2-yl)-3-hydroxypropanenitrile, (R)-1d. Yellow solid (m.p. 107$\left.109{ }^{\circ} \mathrm{C}\right)$, yield 93\%; $[\alpha]_{D}^{25}=+35.4\left(c 1, \mathrm{CHCl}_{3}\right)$, ee $=99 \%$.

(R)-3-(Benzo[b]thiophen-3-yl)-3-hydroxypropanenitrile, $(\boldsymbol{R})-1 \mathrm{e}$. Light yellow oil, yield 83\%; $[\alpha]_{D}^{25}=+69.5\left(c 1, \mathrm{CHCl}_{3}\right)$, ee $=95 \%$.

\section{References}

1. Kamal, A.; Ramesh Khanna, G. B.; Ramu, R.; Krishnaji, T. Tetrahedron Lett. 2003, 44, 4783.

2. Robertson, D. W.; Wong, D. T.; Krushinski, J. H. US Patent 4,956,388, 1990.

3. (a) Paizs, C.; Toşa, M.; Majdik, C.; Tähtinen, P.; Irimie, F. D.; Kanerva, L. T. Tetrahedron: Asymmetry 2003, 14, 619. (b) Paizs, C.; Tähtinen, P.; Toşa, M.; Majdik, C.; Irimie, F. D.; Kanerva, L. T. Tetrahedron 2004, 60, 10533.

4. Itoh, T.; Takagi, Y.; Fujisawa, T. Tetrahedron Lett. 1989, 30, 3811.

5. (a) Itoh, T.; Takagi, Y. Chem. Lett. 1989, 1505. (b) Itoh, T.; Takagi, Y.; Nishiyama, S. J. Org. Chem. 1991, 56, 1521.

6. (a) Kamal, A.; Khanna Ramesh, G. B.; Krishnaji, T.; Ramu, R. Bioorg. Med. Chem. Lett. 2005, 15, 613. (b) Kamal, A.; Khanna Ramesh, G. B.; Ramu, R. Tetrahedron: Asymmetry 2002, 13, 2039.

7. Kamal, A.; Khanna Ramesh, G. B.; Krishnaji, T.; Tekumalla, V.; Ramu, R. Tetrahedron: Asymmetry 2005, 16, 1485.

8. (a) Pàmies, O.; Bäckvall, J.-E. Adv. Synth. Catal. 2001, 343, 726. (b) Pàmies, O.; Bäckvall, J.-E. Adv. Synth. Catal. 2002, 344, 947. 
9. Ma, D.-Y.; Wang, D.-X.; Pan, J.; Huang, Z.-T.; Wang, M.-X. Tetrahedron: Asymmetry 2008, 19, 322.

10. Kamal, A.; Ramesh Khanna, G. B. Tetrahedron: Asymmetry 2001, 12, 405.

11. Kaiser, E. M.; Hauser, C. R. J. Org. Chem. 1968, 33, 3402.

12. Kisanga, P.; McLeod, D.; D Sa, B.; Verkade, J. J.Org. Chem. 1999, 64, 3090.

13. Kawano, Y.; Kaneko, N.; Mukaiyama, T. Chem. Lett. 2005, 34, 1508.

14. (a) Li, X.-G.; Kanerva, L. T. Org. Lett. 2006, 8, 5593. (b) Gedey, S.; Liljeblad, A.; Fülöp, F.; Kanerva, L. T. Tetrahedron: Asymmetry 1999, 10, 2573.

15. (a) Martinelle, M.; Hult, K. Biochim. Biophys. Acta 1995, 1251, 191. (b) García-Alles, L. F.; Gotor, V. Biotechnol. Bioeng. 1998, 59, 163.

16. Sundholm, O.; Kanerva, L.T. J. Chem. Soc., Perkin Trans. 1 1993, 2407.

17. Chen, C.-S.; Fujimoto, Y.; Girdaukas, G.; Sih, C. J. J. Am. Chem. Soc., 1982, 104, 7294. 